platform, and 5 questions were posed to them over the course of 6 weeks in early 2021. The 5 questions focused on (1) what a survivorship service should offer, (2)what resources would participants recommend, (3)what advice would participants give to a newly diagnosed person, (4)what would a successful service look like and (5)what were the participants views on virtual follow up post COVID-19. Responses were text-based.

Result(s)* Of the 16 participants selected, 14 engaged in the process. The overall response rate for all questions was $71 \%$ with 94 individual messages sent over the conversations.

Participants identified that patient needs for survivorship depend on disease site, stage and treatment modalities and do change over time. Nevertheless, they identified areas such as sexual dysfunction, fertility, peer support groups, career and financial advice as essential for any survivorship service to address. All participants spoke of the need for a designated contact person on their medical team whom they can contact with 'minor' queries. The need for psychological support both formal and informal was also highlighted. Participants also spoke about their own unmet needs and a need to increase supports around the issues of menopause, exercise, nutrition and fatigue management.

Participants also felt there were aspects of a survivorship service which could be offered virtually, however, all would prefer some face-to-face contact with their treating team.

Conclusion* The information provided through this digital advisory board will now inform the work of the Living Well Cancer Programme.

\section{PATIENT-REPORTED LOWER LIMB LYMPHEDEMA AND QUALITY OF LIFE AFTER RADICAL SURGERY WITH SENTINEL NODE MAPPING FOR EARLY-STAGE CERVICAL CANCER}

\begin{abstract}
1,2,3 S Sponholtz ${ }^{*},{ }^{4,5} \mathrm{O}$ Mogensen, ${ }^{1,6,7} \mathrm{M}$ Hildebrandt, ${ }^{8} \mathrm{D}$ Schledermann, ${ }^{9} \mathrm{E}$ Parner, ${ }^{10,11} \mathrm{~N}$ Ezendam, ${ }^{10,11} \mathrm{~B}$ De Rooij, ${ }^{2} \mathrm{~A}$ Markauskas, ${ }^{12} \mathrm{~L}$ Froeding, ${ }^{4} \mathrm{~K}$ Fuglsang, ${ }^{4,5} \mathrm{~S}$ Bjørnholt, 1,4,5P Jensen. 'University of Southern Denmark, Institute of Clinical Research, Odense C, Denmark; ${ }^{2}$ Odense University Hospital, Department of Gynaecology and Obstetrics, Odense C, Denmark; ${ }^{3}$ Odense University Hospital and Region of Southern Denmark, Open Patient Data Explorative Network, Odense C, Denmark; ${ }^{4}$ Aarhus University Hospital, Department of Gynaecology and Obstetrics, Aarhus N, Denmark; ${ }^{5}$ Aarhus University, Institute of Clinical Medicine, Aarhus N, Denmark; ${ }^{6}$ Odense University Hospital, Department of Nuclear Medicine, Odense C, Denmark; ${ }^{7}$ Odense University Hospital and University of Southern Denmark, Centre for Innovative Medical Technology, Odense C, Denmark; ${ }^{8}$ Odense University Hospital, Department of Pathology, Odense C, Denmark; ${ }^{9}$ Aarhus University, Department of Public Health, Aarhus C, Denmark; ${ }^{10}$ The Netherlands Comprehensive Cancer Organisation, Amsterdam, Netherlands; ${ }^{11}$ Tilburg School of Social and Behavioral Science, Department of Medical and Clinical Psychology, Tilburg, Netherlands; ${ }^{12}$ Copenhagen University Hospital, Department of Gynaecology, Kbh Ø, Denmark
\end{abstract}

\subsection{6/ijgc-2021-ESGO.577}

Introduction/Background* Sentinel lymph node (SLN) mapping represents a less invasive and more accurate staging technique in women with early-stage cervical cancer, which may reduce or prevent late effects as lymphedema. Despite the increased implementation of SLN mapping, evidence on the potential reduction of lymphedema and its effects on quality of life (QoL) in these women remains scarce. We prospectively evaluated patient-reported lower limb lymphedema and QoL in women with early-stage cervical cancer undergoing radical surgery with SLN mapping.

Methodology In a national multi-institutional study, we included women with early-stage cervical cancer from March
2017-January 2021 to undergo radical surgery including SLN mapping. Women with tumours $>20 \mathrm{~mm}$ underwent completion pelvic lymphadenectomy (PL). The incidence and severity of lymphedema and QoL were evaluated using validated patient-reported outcome measures before surgery and three months postoperative. Changes over time were investigated using linear regression.

Result(s)* Two hundred of 245 (81.6\%) included women completed the baseline and three-month questionnaires. The incidence of lymphedema was $7.2 \%$ versus $31.5 \%$ in women who underwent SLN mapping alone and completion PL, respectively ( $p<0.001)$. Lymphedema scores in the leg, genital, and groin were affected in both groups, but significantly more after PL. The differences between groups remained significant in a multivariate analysis adjusting for, e.g., adjuvant therapy and age. PL significantly affected the severity of lymphedema regarding physical performance $(\mathrm{p}=0.001)$, appearance $(\mathrm{p}=$ $0.008)$, besides heaviness, weakness, and pain in the legs $(\mathrm{p}<$ 0.001). Lymphedema was negatively associated with impaired body image $(\mathrm{p}=0.002)$, physical $(\mathrm{p}=0.008)$, role $(\mathrm{p}=$ $0.04)$, and social functioning $(\mathrm{p}=0.007)$ and a higher level of fatigue $(\mathrm{p}=0.01)$.

Conclusion* SLN mapping combined with PL is associated with a significantly higher incidence and more severe lymphedema three months postoperatively than SLN mapping alone. Lymphedema was associated with lower QoL in several domains.

\section{CAN PHARMACOGENETICS PREDICT CHEMOTHERAPY INDUCED PERIPHERAL NEUROPATHY IN GYNECOLOGIC CANCER PATIENTS TREATED WITH TAXANE-BASED CHEMOTHERAPY?}

${ }^{1} \mathrm{~K}$ Dorr*, ${ }^{1} \mathrm{~A}$ Ward, ${ }^{1} \mathrm{D}$ Mysona, ${ }^{2} \mathrm{E}$ Shaver, ${ }^{1} \mathrm{~B}$ Rungruang, 'S Ghamande. 'Augusta University Medical Center, Augusta, USA; ${ }^{2}$ Consultative Genomics, Peachtree Corners, USA

\subsection{6/ijgc-2021-ESG0.578}

Introduction/Background* Taxane-based chemotherapy used in treating gynecologic malignancies, results in one third of patients developing grade 2 or higher chemotherapy induced peripheral neuropathy (CIPN). Currently, there is no test which predicts who is at increased risk of CIPN. Pharmacogenomic testing may result in the ability to better predict risk of CIPN allowing personalized treatments to avoid this distressing side effect and improve quality of life.

Methodology Patients were enrolled from August 2020 to November 2020 in a prospective, case-control trial evaluating pharmacogenetic predictors of CIPN in women previously treated with at least 3 cycles of taxane-based chemotherapy for histologically confirmed gynecologic malignancies. Buccal saliva samples were used to test for 64 prespecified drug metabolism variations. All testing was performed by $\alpha$ LPHAGENOMIX laboratories.

Result(s)* Of 102 enrolled patients, 58\%, 28\%, 14\%, and 2\% had ovarian, endometrial, or cervical cancers, respectively. The median age was 67 , and $72 \%$ were white. A total of $16 \%$ of patients were treated with 3-4 cycles, 57\% received 5-7 cycles, and $27 \%$ received 8 or more cycles of chemotherapy. Half of the patients had grade 2 CIPN. There was no difference in age, race, disease site, or number of chemotherapy cycles ( $p>0.05$ ) when comparing between the two groups. Of the 64 tested pharmacogenomic alterations, CYP3A5 genotype 